\title{
Correction
}

\section{Correction: Sharma et al., Neurosilence: Profound Suppression of Neural Activity following Intracerebral Administration of the Protein Synthesis Inhibitor Anisomycin}

In the article "Neurosilence: Profound Suppression of Neural Activity following Intracerebral Administration of the Protein Synthesis Inhibitor Anisomycin" by Arjun V. Sharma, Frank E. Nargang, and Clayton T. Dickson, which appeared on pages 2377-2387 of the February 15, 2012 issue, the authors regret a citation error in the Discussion on page 2385. In the last phrase of the first paragraph in the subsection entitled "Can neural inactivation caused by protein synthesis inhibition explain prior behavioral results?" the citation to Bidenkapp et al. (2009) is incorrect. It should read Sparks et al. (2011). The full citation for this article is: Sparks FT, Lehmann H, Sutherland RJ (2011) Between-systems memory interference during retrieval. Eur J Neurosci 34:780-786.

DOI: 10.1523/JNEUROSCI.2572-12.2012 